\title{
Condições sanitárias e avaliação dos procedimentos de biossegurança adotados em estúdios de tatuagem
}

\section{Health conditions and evaluation of biosafety procedures adopted in tattoo studies}

Brena Mesquita Santos

Marcelle Melo Magalhães (iD)

Francisco Vassiliepe Sousa Arruda iD

Mauro Vinicius Dutra Girão* iD

Centro Universitário Inta (Uninta), Sobral, CE, Brasil

\section{* E-mail: viniciusgirao@gmail.com}

Recebido: 26 jun 2020

Aprovado: 08 out 2020

\begin{abstract}
RESUMO
Introdução: 0 procedimento de tatuagem oferece risco biológico aos profissionais e clientes. Objetivo: Descrever as condições higiênicas e sanitárias, bem como analisar a adoção de procedimentos para a gestão de riscos de biossegurança por profissionais tatuadores que atuam em estúdios de tatuagem de um município localizado na região noroeste do estado do Ceará, Brasil. Método: Estudo qualitativo, descritivo e transversal, realizado em cinco estúdios de tatuagem através da aplicação de um checklist baseado em Referência Técnica para o funcionamento dos serviços de tatuagem e piercing da Agência Nacional de Vigilância Sanitária (2009). Resultados: Os principais pontos negativos fazem alusão ao funcionamento de estúdios clandestinos, registro precário dos dados pessoais dos clientes e coleta inadequada do lixo infectante. Por outro lado, os principais pontos positivos alcançados nesse estudo estão relacionados com o descarte adequado de objetos perfurocortantes, existência de local reservado e adequado para o procedimento de tatuagem, incluindo pia para a lavagem das mãos próxima ao local do procedimento. A pesquisa evidenciou também que os profissionais tatuadores possuem materiais e equipamentos apropriados para as respectivas práticas, porém executam procedimentos de biossegurança de maneira incorreta, os quais predispõem profissionais e clientes a fatores de risco biológico. Conclusões: Os estúdios de tatuagem analisados apresentam pobre adequação às normas da Vigilância Sanitária, as quais são insuficientes para garantir a promoção e manutenção da segurança para a saúde de todos os envolvidos em procedimentos de estética corporal do tipo tatuagem.
\end{abstract}

PALAVRAS-CHAVE: Gestão de Riscos; Vigilância Sanitária; Gestão da Segurança; Fatores de Risco; Risco à Saúde Humana

\section{ABSTRACT}

Introduction: The tattoo procedure offers biological risk to professionals and clients. Objective: To describe the hygienic and sanitary conditions, as well as to analyze the adoption of procedures for the management of biosafety risks by professional tattoo artists who work in tattoo studios in a municipality located in the Northwestern Region of the State of Ceará, Brazil. Method: Qualitative, descriptive and transversal study, carried out in five tattoo studios through the application of a checklist based on the Technical Standard for the operation of tattoo and piercing services by the Agência Nacional de Vigilância Sanitária (2009). Results: The main negative points allude to the functioning of clandestine studios, poor registration of personal data of customers and inadequate collection of infectious waste. On the other hand, the main positive points achieved in this study are related to the proper sharps waste, the existence of a reserved and adequate place for the tattoo procedure, including a sink for washing hands close to the procedure site. The research also showed that professional tattoo artists have appropriate materials and equipment for their respective practices, but perform biosafety procedures incorrectly, which predispose professionals and clients to biological risk factors. Conclusions: The tattoo studios analyzed have poor compliance with health surveillance norms, which are insufficient to guarantee the promotion and maintenance of safety for the health of everyone involved in body aesthetic procedures such as tattoos.

KEYWORDS: Risk Management; Health Surveillance; Safety Management; Risk Factors; Health Risk 


\section{INTRODUÇÃO}

A palavra tatuagem é de origem polinésia'. Trata-se de um procedimento invasivo de decoração corporal de caráter estético com o objetivo de pigmentar a pele por meio da introdução de substâncias corantes, com o uso de agulhas. 0 tatuador é a pessoa que domina as técnicas de pigmentação exógena implantada na camada dérmica da pele, que devem ser realizadas em locais apropriados denominados estúdios de tatuagem. Os procedimentos realizados nestes locais devem seguir um padrão rigoroso de higienização para que não exponha os clientes a riscos sanitários ${ }^{2}$.

Atualmente, a prática da tatuagem tem aumentado entre os brasileiros. No Brasil, o número de estúdios de tatuagem teve aumento de $24,1 \%$ entre 2016 e 2017 . É um procedimento estético comum entre diversas classes sociais e idades, predominantemente feito por pessoas na faixa etária de 19 a 25 anos ( $48,2 \%$ dos tatuados de ambos os gêneros), sendo que $59,9 \%$ delas são mulheres ${ }^{3}$.

Por se tratar de um procedimento invasivo, essa prática oferece risco biológico aos profissionais e clientes, possibilitando a transmissão de doenças infectocontagiosas. Em adição, o procedimento pode desencadear alergias e intoxicação por meio dos materiais e equipamentos utilizados que entram em contato com o sangue. É fato que a frequência de infecção associada a procedimentos de tatuagem vem aumentando nos últimos anos, podendo ser decorrente da identificação mais eficiente do agente etiológico, da popularidade das tatuagens ou das práticas dos procedimentos serem insatisfatórios do ponto de vista sanitário. Assim, pode ocorrer transmissão de doenças devido a material contaminado, o que constitui um importante problema de saúde pública ${ }^{4,5,6}$.

Desta forma, devem ser levadas em conta as práticas adequadas de biossegurança, que, segundo Cheuhuen $\mathrm{Neto}^{7}$, são um conjunto de ações destinadas a prevenir, controlar, reduzir ou eliminar riscos inerentes às atividades que possam comprometer a saúde humana, animal e o meio ambiente e têm como principal objetivo contribuir para a qualidade dos produtos e dos serviços, ao proteger os indivíduos envolvidos no decorrer das atividades profissionais.

Pela prática da tatuagem ser considerada um serviço de interesse à saúde de características particulares, a Agência Nacional de Vigilância Sanitária (Anvisa)² produziu uma referência técnica para o funcionamento dos serviços de tatuagem para diminuir e/ou prevenir riscos e proteger a saúde das pessoas que desejam ser tatuadas.

Para alcançarem condições satisfatórias no serviço prestado aos clientes, os profissionais tatuadores devem desenvolver ações que sejam capazes de eliminar, diminuir ou prevenir riscos e intervir nos problemas sanitários decorrente da prestação desses serviços, controlando as condições ambientais e os procedimentos de tatuagem e, consequentemente, promovendo a adesão às normas de biossegurança $a^{2,4}$.
Assim, as ações de orientação, consultorias e educação permanente quanto à biossegurança no ofício de tatuar podem apontar uma nova área de atuação para os profissionais de saúde, visto que, entre suas competências, se destacam a educação em saúde e o treinamento de pessoas em proteção da saúde do trabalhador ${ }^{5,8}$.

O presente estudo se justifica devido à prática da tatuagem estar disseminada em todas as camadas da população e os procedimentos adotados poderem influenciar na frequência de infecção, por isso, torna-se necessário investigar as práticas de biossegurança em estúdios de tatuagem como um tema emergente de interesse à saúde. Assim, o presente trabalho foi conduzido baseado na seguinte pergunta norteadora: "Qual a situação higiênico-sanitária e o nível de biossegurança de estúdios de tatuagem e os possíveis riscos aos quais os clientes e trabalhadores estão expostos?".

Esta pesquisa teve por objetivo analisar o conhecimento e a adoção de procedimentos de biossegurança adotados pelos profissionais tatuadores em estúdios de tatuagem de um município localizado na região noroeste do estado do Ceará, classificar e descrever as condições higiênico-sanitárias dos estúdios de tatuagem, listando as irregularidades encontradas.

\section{MÉTODO}

Trata-se de um estudo de campo com abordagem qualitativa, descritiva e transversal que se baseia no preenchimento de checklist durante a coleta de dados nos estúdios de tatuagem, evidenciando os riscos de saúde que os clientes estão expostos.

Para a realização do estudo, inicialmente foi adquirida junto à Vigilância Sanitária municipal e por redes sociais a lista dos estúdios de serviço de tatuagem em atividade no município. Em seguida, os profissionais tatuadores foram contatados por telefone e redes sociais, com o intuito de adquirir o Termo de Anuência. Com a posse dos Termos de Anuência, estes foram adicionados ao projeto que foi submetido ao Comitê de Ética e Pesquisa do Centro Universitário INTA (Uninta) e recebeu o parecer favorável número 3.028.256/2018.

Assim, o estudo foi realizado com profissionais tatuadores e seus estúdios de tatuagem instalados em um município da região noroeste do estado do Ceará, durante o mês de novembro de 2018.

Após esclarecimento sobre os objetivos do estudo e a importância da participação, foi assinado o Termo de Consentimento Livre e Esclarecido (TCLE) com a aceitação do profissional tatuador em participar do estudo. Em seguida, realizou-se a inspeção, sendo que o nome do município, dos estúdios, endereço e profissional tatuador foram omitidos a fim de preservar a identidade dos participantes. Quando necessário, esses dados foram substituídos por letras do alfabeto. 
A coleta de dados foi realizada obtendo-se dados dos profissionais tatuadores referentes a gênero, idade, tempo que exercem a atividade, participação em curso de capacitação, atualização e conhecimento sobre doenças infectocontagiosas. Em seguida, por meio de checklist baseado no manual de referência técnica para o funcionamento dos serviços de tatuagem da Anvisa², os estúdios de tatuagem foram analisados. 0 instrumento de coleta de dados era composto por questões avaliativas fechadas referentes a cinco critérios: 1) Aquisição e armazenamento de dados dos clientes, composto por nove itens; 2) Estrutura física, composto por 15 itens; 3) Materiais e equipamentos, composto por três itens; 4) Boas práticas individuais, composto por sete itens; e 5) Gerenciamento de resíduos, composto por cinco itens; totalizando 39 questões. Cada estúdio teve apenas uma coleta de dados ao longo do período de estudo.

A coleta das informações ocorreu em horários convenientes para cada profissional tatuador e sem a presença de clientes, a fim de não ocasionar constrangimento aos profissionais devido a haver a possibilidade de identificação de não conformidades no ambiente de trabalho e nos procedimentos realizados.

Após o preenchimento do checklist baseado no manual de referência técnica para o funcionamento dos serviços de tatuagem da Anvisa $^{2}$, os estúdios de tatuagem foram classificados em três níveis de satisfação dos critérios: satisfaz completamente (SC), satisfaz parcialmente (SP) e não satisfaz (NS). 0 primeiro nível é alcançado quando o serviço de tatuagem atende $100 \%$ dos critérios da Anvisa, o segundo, quando atende $1 \%-99 \%$ e o terceiro, quando não atende nenhum. Estes critérios foram desenvolvidos pelos autores da pesquisa por não haver instrumento para classificar a qualidade das práticas de biossegurança adotadas em estúdios de tatuagem.

\section{RESULTADOS E DISCUSSÃO}

A lista dos estúdios de serviço de tatuagem em atividade no município trazia dez estabelecimentos. É oportuno ressaltar que a Vigilância Sanitária municipal tinha registro de apenas três estúdios de tatuagem, ou seja, sete estabelecimentos realizavam a atividade sem possuir o alvará sanitário. Nossos resultados apresentam a realidade evidenciada em metade dos estúdios de tatuagem em atividade no município, pois cinco profissionais tatuadores não aceitaram participar da pesquisa.

Dos cinco estúdios de tatuagem onde houve coleta de dados, apenas um possuía cadastro junto à Vigilância Sanitária municipal (estúdio D). Todos os profissionais tatuadores eram do gênero masculino e tinham idade entre 23 e 40 anos. Quanto ao tempo de profissão, três dos participantes da pesquisa possuíam menos de cinco anos na atividade e dois, mais de dez anos na atividade.

Em relação aos níveis de satisfação dos estúdios de tatuagem quanto aos critérios da referência técnica para o funcionamento dos serviços de tatuagem segundo a Anvisa ${ }^{2}$ os resultados mostraram que os estúdios $A, B$ e $C$ não cumpriram o critério 1. $O$ estúdio $D$ foi o único que possuía alvará sanitário, satisfazendo parcialmente ou completamente os quesitos presentes em cada critério, visto que os demais estúdios apresentaram pontos de não satisfação aos critérios (Quadro).

Estudo semelhante ${ }^{9}$ também verificou que os profissionais de estética eram adultos jovens com relativo tempo de atuação na área e que muitos estabelecimentos de estética funcionam sem cadastro junto à Vigilância Sanitária municipal, mas o estudo não encontrou diferenças entre os estabelecimentos cadastrados e os não cadastrados. Fato que difere dos nossos achados.

Dentre as irregularidades sanitárias identificadas, a falta do alvará sanitário foi frequente. A ausência desse documento compromete a qualidade do serviço oferecido ao cliente, já que muitas irregularidades só são identificadas durante as inspeções e as ações de controle sanitário.

Possivelmente a falta de alvará é um dos motivos para o grande número de não conformidades encontradas na nossa amostra, o que valoriza o trabalho da Vigilância Sanitária quanto às exigências sanitárias a serem adotadas pelos profissionais que requerem o alvará sanitário de funcionamento.

A seguir serão apresentados e discutidos os critérios: aquisição e armazenamento de dados dos clientes (critério 1); estrutura física (critério 2); materiais e equipamentos (critério 3); boas práticas individuais (critério 4) e gerenciamento de resíduos (critério 5), previamente estabelecidos (Figura 1 e 2).

Quadro. Satisfação das conformidades dos estúdios de tatuagem aos critérios da referência técnica para o funcionamento dos serviços de tatuagem da Agência Nacional de Vigilância Sanitária².

\begin{tabular}{|lcccccc|}
\hline Estúdio & \multicolumn{7}{c}{ Critérios } \\
\cline { 2 - 7 } & Alvará sanitário & $\begin{array}{c}\text { Aquisição e } \\
\text { armazenamento de } \\
\text { dados dos clientes }\end{array}$ & Estrutura física & $\begin{array}{c}\text { Materiais e } \\
\text { equipamentos }\end{array}$ & $\begin{array}{c}\text { Boas práticas } \\
\text { individuais }\end{array}$ & $\begin{array}{c}\text { Gerenciamento de } \\
\text { resíduos }\end{array}$ \\
\hline A & NÃO & NS & SP & SC & SP & SP \\
B & NÃO & NS & SP & SC & SP & SP \\
C & NÃO & NS & SP & SC & SP & SC \\
D & SIM & SP & SP & SC & SP & SC \\
E & NÃO & SP & SP & SC & SP & SP \\
\hline
\end{tabular}

Fonte: Elaborado pelos autores, 2020.

NS: não satisfaz; SP: satisfaz parcialmente; SC: satisfaz completamente. 


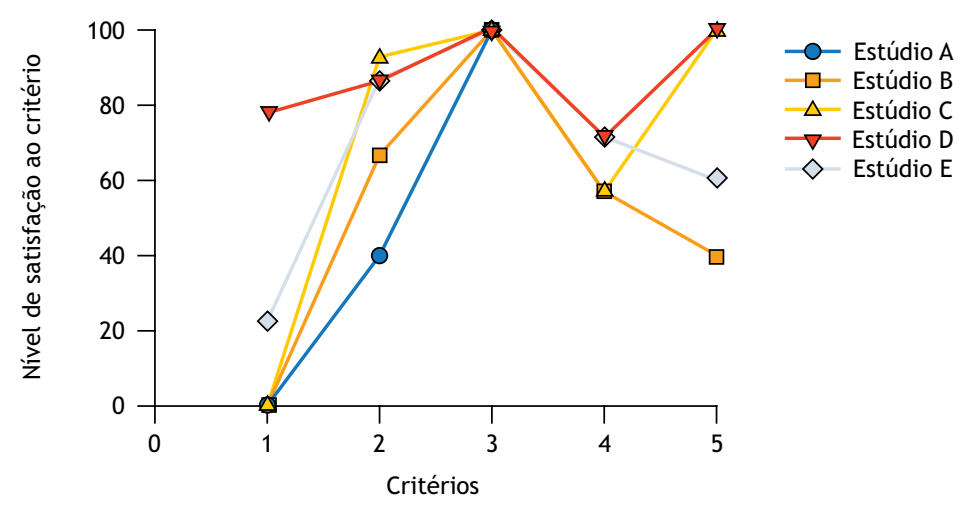

Fonte: Elaborada pelos autores, 2020.

Figura 1. Nível de satisfação global dos estúdios de tatuagem aos critérios da referência técnica para o funcionamento dos serviços de tatuagem da Agência Nacional de Vigilância Sanitária².

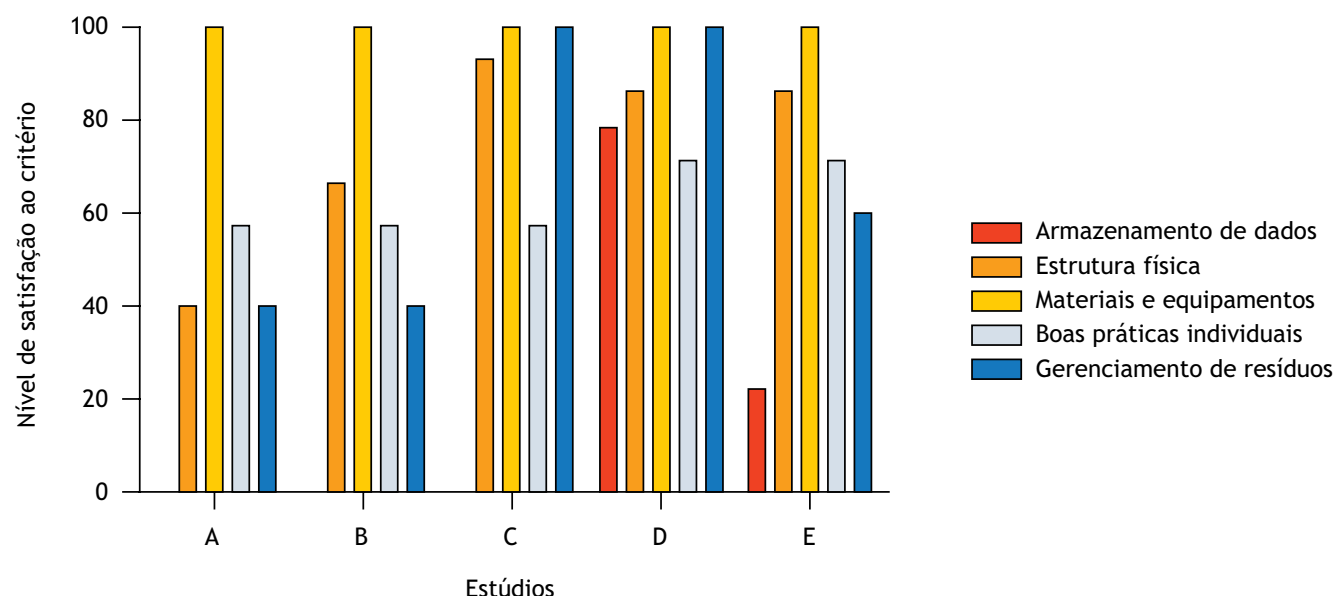

Fonte: Elaborada pelos autores, 2020.

Figura 2. Nível de satisfação de cada estúdio de tatuagem aos critérios de armazenamento de dados, estrutura física, materiais e equipamentos, boas práticas e gerenciamento de resíduos segundo a referência técnica para o funcionamento dos serviços de tatuagem².

Aquisição e armazenamento de dados dos clientes

Nenhum estúdio satisfez completamente o checklist, pois não há registro sobre possíveis alergias e informações vacinais referentes ao cliente, o que poderia diminuir o risco de complicações. Os profissionais tatuadores estão sujeitos a um elevado grau de risco ocupacional, destacando-se a exposição aos agentes biológicos. As principais doenças passíveis de serem transmitidas por meio da interação cliente/profissional são: a hepatite C (HCV), a hepatite $B(\mathrm{HBV})$ e a síndrome da imunodeficiência humana adquirida (AIDS) ${ }^{10}$.

A hepatite B é a doença infecciosa que mais acomete os profissionais que utilizam materiais perfurocortantes. Ela pode ser transmitida através de contato com solução de continuidade, por meio da pele não íntegra e mucosas, e representa um sério problema de saúde pública, devido ao número elevado de indivíduos portadores da doença e as complicações decorrentes de sua evolução ${ }^{11}$.
A imunização é a principal forma de prevenção e torna-se indispensável entre tatuadores e clientes, devido à exposição a materiais biológicos. A existência dos testes rápidos ainda é um método pouco utilizado e até desconhecido por estes profissionais ${ }^{12}$.

A tatuagem, por ser um ato traumático, desencadeia uma reação inflamatória inespecífica passageira e discreta logo que a agulha começa a perfurar a pele. Além disso, os pigmentos de tatuagem não permanecem inertes na derme, pois primeiramente ocorre a sua fagocitose pelos macrófagos, células endoteliais e fibroblastos e, posteriormente, a ativação de macrófagos. Discretas alterações inflamatórias são observadas anos após a realização do procedimento de tatuagem, como, por exemplo, tentativas de degradar o material estranho. Nos indivíduos que fazem tatuagens extensas, pode haver linfadenomegalia. Complicações ocorrem em aproximadamente $2 \%$ dos casos, incluindo infecções, neoplasias e dermatoses inflamatórias ${ }^{13}$. 


\section{Estrutura física}

Nenhum dos estúdios satisfez esse quesito. Dentre os motivos estão a inadequação quanto ao piso, ao teto e à parede. Os estúdios não possuíam sala de procedimento com pisos, tetos e paredes laváveis e pia para higienização das mãos no local de realização do procedimento de tatuagem. Estas inadequações por parte dos estúdios que compuseram a amostra deste estudo estão relacionadas a eles funcionarem clandestinamente na residência dos profissionais.

Foi detectado que a maioria dos estúdios $(n=4)$ dispunha de local reservado adequado para o procedimento de tatuagem. A Anvisa ${ }^{2}$ preconiza que esta prática seja realizada em ambiente exclusivo, sem presença de outros clientes, para diminuição de riscos e maior privacidade e conforto para o cliente. Devido à prática da tatuagem ser um procedimento invasivo, é imprescindível a realização de lavagem das mãos com técnica adequada. Foi detectado que boa parte dos profissionais $(n=3)$ dispunham de pia para a lavagem das mãos próxima ao local de atendimento ao cliente e os demais usavam a pia do banheiro.

Apesar de todos os entrevistados afirmarem lavar as mãos entre um cliente e outro, a inexistência de uma pia próxima ao local de atendimento sugere que essa prática não esteja sendo realizada de forma adequada.

A higiene das mãos (HM) é amplamente reconhecida como uma das principais estratégias para a prevenção das infecções. 0 termo HM engloba a higiene simples e a higiene antisséptica ${ }^{14}$.

Segundo Anacleto, Peterline e Pedreira ${ }^{15}$, a HM é uma intervenção de baixo custo e com indicações sustentadas por fundamentação científica. Há anos existem evidências de que as mãos dos profissionais constituem o principal vetor de transmissão de microrganismos patogênicos e que higienizá-las contribui significativamente para reduzir a incidência de infecções. No entanto, a adesão às práticas recomendadas de HM é de difícil alcance e sustentação.

Diversos serviços de saúde não estão atendendo plenamente às normas preconizadas quanto a estruturas física ${ }^{16}$ e precisam de um gerenciamento adequado para que ofertem serviços de forma que as necessidades de saúde da população sejam atendidas. Para isso é necessário investimento, planejamento e organização de processos de trabalho ${ }^{17}$.

\section{Materiais e equipamentos}

Este critério aborda se as marcas das tintas são autorizadas pela Anvisa, se são fracionadas para o uso, descartadas adequadamente e se os materiais de uso são descartáveis. Todos os estúdios satisfizeram esse critério, visto que os tatuadores participantes do estudo fazem uso de tintas aprovadas pela Anvisa e dentro do prazo de validade, além de fazerem uso de material estéril e descartável, havendo dispensa de autoclave.

Segundo a Anvisa ${ }^{2}$, mesmo utilizando tintas aprovadas o tatuador deve fracionar a tinta utilizada para cada cliente, desprezar o que sobrar, minimizando assim o risco de contaminação entre clientes. É importante a regulação e a fiscalização sobre as tintas comercializadas. Não devem ser usadas fora do prazo de validade e, quando houver a presença de mercúrio em sua composição, devem ter seu uso limitado. As seguintes marcas são autorizadas e regulamentadas: Starbrite Colors $\odot$, Iron Works $\odot$ e Eletric ink@.

Deve ser dada atenção aos pigmentos presentes nas tintas utilizadas para realização da tatuagem devido a fato de serem um frequente motivo de consultas médicas, pois há alto teor de derivados de mercúrio, o que causa alergia no cliente. Entre as complicações ocasionadas pela prática da tatuagem, podem ser mencionadas: dermatoses, dermatite de contato, queloides, entre outras ${ }^{18}$.

\section{Boas práticas individuais}

Em relação à capacitação, quatro dos entrevistados afirmaram ter realizado curso profissionalizante ou de atualização. Dentre os pesquisados, todos afirmaram saber o que é a AIDS e o seu modo de transmissão, e apenas um não reconheceu o que é hepatite e sua transmissão.

No Brasil, a capacitação profissional não é um critério utilizado para liberação de alvarás sanitários. Os profissionais tatuadores são autônomos não têm obrigatoriedade de possuir formação na área de saúde. 0 exercício desta atividade não requer formação específica e nenhuma qualificação, o que sugere a oferta de treinamento por um profissional da área da saúde, para que profissionais tatuadores passem a saber lidar com os riscos e a biossegurança ${ }^{4,5}$.

Entretanto, evidências mostraram ${ }^{5,19,20,21}$ que profissionais com menor grau de escolaridade apresentaram conhecimento inadequado sobre agentes infectocontagiosos, menor frequência de imunização, estão mais sujeitos a acidentes com material perfurocortante ou com exposição ao material biológico. Tendo conhecimento sobre os riscos de contaminação, estes apresentam maior preocupação com a AIDS e as hepatites B e C.

Estados Unidos, Europa e Oceania possuem normatização abrangente que contempla a formação dos tatuadores, incluindo a educação continuada obrigatória e a aplicação de um exame escrito. Os tatuadores devem seguir protocolos de higiene, capacitação e educação em saúde e ter os estúdios monitorados. Devido ao risco de transmissão de doenças por meio dessas práticas, muitos países têm desenvolvido regulamentos visando o controle de infecção e a prevenção de doenças relacionadas aos serviços de tatuagem ${ }^{22,23}$. Práticas como essas poderiam ser adotadas pelos órgãos fiscalizadores brasileiros.

No Brasil, a Norma Regulamentadora (NR) $\mathrm{n}^{\circ} 32$, de 11 de novembro de $2005^{24}$, objetiva estabelecer diretrizes básicas para implementar medidas de proteção à segurança e à saúde dos trabalhadores. Dentre as normas estabelecidas são preconizados: o uso de equipamentos de proteção individual (EPI), a higienização das mãos e a vacinação contra hepatite $\mathrm{B}$, tétano e difteria. A NR $\mathrm{n}^{\circ} 6$, de 8 de junho de $1978^{25}$, considera EPI todo dispositivo ou produto de uso individual utilizado 
pelo trabalhador, destinado à proteção de riscos suscetíveis de ameaçar a segurança e a saúde no trabalho. Porém, mesmo com a adoção das medidas de proteção, ainda ocorrem acidentes com materiais perfurocortantes. Sendo assim, todos os envolvidos na atividade devem adotar medidas para prevenir acidentes de trabalho ${ }^{10,19,20,21}$.

Os princípios, as diretrizes e as estratégias para o desenvolvimento da atenção integral à saúde do trabalhador são definidos com ênfase na vigilância, visando a promoção e a proteção da saúde dos trabalhadores, assim como a redução da morbimortalidade decorrente dos modelos de desenvolvimento e dos processos produtivos ${ }^{26}$.

A realização de estudos epidemiológicos que foquem na ocorrência dos acidentes de trabalho com material biológico poderá contribuir para aumentar a compreensão sobre os processos determinantes para a ocorrência dos acidentes, assim como gerar subsídios para novas pesquisas e programas de intervenção em outros contextos sociais. Pois ainda carece de estudos para serem utilizados como instrumento de conhecimento e de trabalho, para os profissionais e instituições ${ }^{27}$.

Nesse sentido, os acidentes de trabalho com material biológico potencialmente contaminado ainda são frequentes e podem acarretar sérias consequências de ordem física e psicossocial à vítima, e judiciais às instituições de saúde.

Transmissões de doenças devido a material contaminado constituem um importante problema de saúde pública. Essas infecções podem permanecer de forma assintomática ou se manifestar por meio de sinais e sintomas. Os adolescentes e jovens são considerados grupos vulneráveis. 0 risco se intensifica quando os adolescentes estão expostos e em situações de vulnerabilidade, como os que fazem tatuagens em locais inadequados e não cadastrados na Vigilância Sanitária ${ }^{6,28}$.

Outro estudo ${ }^{5}$ chama atenção para o fato de que o profissional tatuador tem como principal preocupação a arte de tatuar e que, por diversas vezes, pode deixar para segundo plano os cuidados com biossegurança.

\section{Gerenciamento de resíduos}

Todos os estúdios dispunham de recipiente rígido resistente à punção para o descarte de agulhas e objetos perfurocortantes e dois dispunham de coleta adequada para o lixo infectante. Três deles entregam, respectivamente, em Hospital, Clínica Veterinária e Estratégia de Saúde da Família (ESF) e os demais contratam uma empresa para coleta. Apenas dois dos estúdios possuem Plano de Gerenciamento de Resíduos de Saúde (PGRS) e contrato com empresa adequada para realização do descarte correto dos resíduos.

Por sua vez, a Resolução da Anvisa $\mathrm{n}^{\circ} 222$, de 28 de março de $2018^{29}$, que dispõe sobre as boas práticas de gerenciamento dos resíduos de serviços de saúde, apresenta um conjunto de procedimentos de gestão, planejados e implementados a partir de bases científicas e técnicas, normativas e legais, com o objetivo de minimizar a produção de resíduos e proporcionar aos resíduos gerados um encaminhamento seguro, visando a proteção dos trabalhadores, a preservação da saúde pública, dos recursos naturais e do meio ambiente. 0 gerenciamento de resíduos serviços de saúde, inclusive estúdios de tatuagem, deve abranger todas as etapas de planejamento dos recursos físicos, dos recursos materiais e da capacitação dos recursos humanos envolvidos para ao condicionamento e disposição final dos resíduos.

Mas o que vem sendo constatado é que os profissionais de estúdios de tatuagem, mesmo exercendo atividades de serviço de saúde, muitas vezes negligenciam o gerenciamento de seus resíduos ${ }^{30}$.

É importante enfatizar que todos os materiais descartados em ambiente de saúde representam grande risco à saúde. Portanto, deve-se ter consciência e responsabilidade socioambiental ao manuseá-los e descartá-los, cumprindo o plano de gerenciamento de resíduos sólidos de saúde e contribuindo para a segurança dos profissionais, do público atendido e do meio ambiente ${ }^{31}$.

É necessária a adoção de medidas preventivas voltadas aos clientes, como a confecção e a utilização de cartazes informativos nos estúdios de tatuagem sobre os possíveis riscos e cuidados posteriores à realização deste procedimento estético, para o cliente obter o conhecimento necessário e estar apto a prevenir riscos e complicações ao escolher realizar o procedimento. Bem como a adoção de medidas preventivas voltadas aos proprietários dos estúdios de tatuagem, como a criação e o preenchimento de um livro de ocorrência, para registro de acidentes de trabalho, os quais devem ser notificados no momento que procurarem atendimento médico.

\section{CONCLUSÕES}

O fato mais alarmante constatado na realidade estudada foi o número considerável de estúdios de serviço de tatuagem que funcionam sem alvará sanitário. Destaca-se que as limitações do presente estudo decorrem do fato de os trabalhadores informais, aqueles que não possuem alvará ou atendem em seu próprio domicílio em um ambiente de trabalho improvisado, apresentaram resistência a esta pesquisa. A coleta de dados sendo realizada nestes estabelecimentos poderia mostrar outra realidade e possibilitar outras conclusões.

Entretanto, os estúdios inspecionados, mesmo com suas limitações sanitárias, satisfazem parcialmente ou completamente os critérios estabelecidos para o funcionamento dos serviços de tatuagem quanto à estrutura física; materiais e equipamentos; boas práticas individuais e gerenciamento de resíduos. 0 único critério com total desacordo é referente à aquisição e ao armazenamento de dados pessoais dos clientes, o que pode influenciar na transmissão de doenças ou no desenvolvimento de reações adversas ao procedimento. Em relação a reações alérgicas às tintas para a pigmentação da pele, os estúdios estão de acordo com a regulamentação sanitária brasileira.

Embora esses resultados sejam encorajadores, o nível de adequação as normas da Vigilância Sanitária são insuficientes para 
garantir a promoção e a manutenção da saúde segura para todos os envolvidos no procedimento de estética corporal do tipo tatuagem.

Foi evidenciado que, mesmo com boa escolaridade, relativo tempo de profissão e realização de cursos de capacitação, alguns procedimentos de biossegurança vêm sendo realizados erroneamente pelos profissionais. Isso evidencia que apenas a capacitação sem fiscalização não garante o cumprimento das boas práticas de biossegurança. Deve haver maior conscientização por meio da educação sanitária promovida pelos órgãos competentes voltada para estes profissionais sobre a transmissão e prevenção de doenças infecciosas, o que pode contribuir positivamente na transformação dos comportamentos de risco à saúde durante o exercício profissional.

Os setores de vigilância em saúde municipais e estaduais podem adotar a referência técnica para o funcionamento de serviços de tatuagem para elaborar e instituir legislações como mecanismos de avaliação, orientação e fiscalização as práticas de estética corporal em estúdios de tatuagem, a fim de que garantam segurança aos tatuadores e clientes envolvidos. Bem como a criação e a padronização de um instrumento do tipo checklist para utilização nas vistorias da Vigilância Sanitária municipais, visto que muitas cidades não possuem instrumento para regulação e liberação de alvará sanitário.

\section{REFERÊNCIAS}

1. Urdang M, Mallek JT, Mallon WK. Tattoos and piercings: a review for the emergency physician. West J Emerg Med. 2011;12(4):393-8. https://doi.org/10.5811/westjem.2011.4.2268

2. Agência Nacional de Vigilância Sanitária - Anvisa. Referência técnica para o funcionamento dos serviços de tatuagem e piercing. Brasília: Agência Nacional de Vigilância Sanitária; 2009[acesso 18 maio 2018]. Disponível em: http://portal.anvisa.gov.br/

3. Serviço Brasileiro de Apoio às Micro e Pequenas Empresas Sebrae. Estúdios de tatuagem. São Paulo: Serviço Brasileiro de Apoio às Micro e Pequenas Empresas; 2017[acesso 4 dez 2018]. Disponível em: http://www.bibliotecas. sebrae.com.br/chronus/ARQUIVOS_CHRONUS/bds/ bds.nsf/3F64D1DDA8D8F56A832578A300516767/\$File/ NT000457C2.pdf.

4. Diniz AF, Matté GR. Procedimentos de biossegurança adotados por profissionais de serviço de embelezamento. Saúde Soc. 2013;22(3):751-9. https: / / doi.org/10.1590/S0104-12902013000300009

5. Sousa KS, Martins ERC, Costa CMA, Spindola T, Ramos RCA, Barros AS. Saberes e práticas de biossegurança entre tatuadores: uma contribuição do enfermeiro. Rev Enferm UERJ. 2016;4(24):1-6. https://doi.org/10.12957/reuerj.2016.23694

6. Carvalho PMNS, Guimarães RA, Moraes PA, Teles SA, Matos MA. Prevalência de sinais e sintomas e conhecimento sobre doenças sexualmente transmissíveis. Acta Paul Enferm. 2015;28(1):95-100. https://doi.org/10.1590/1982-0194201500016

7. Cheuhuen Neto JA, Lima MG, Santos JLCT, Costa LA, Estevanin GM, Freire MR et al. Conhecimento e adesão as práticas de biossegurança entre estudantes da área da saúde. Braz J Surg Clin Res. 2017;21(2):82-7.

8. Silva FJCP, Santos PCF, Reis FP, Lima SO. Estado vacinal e conhecimento dos profissionais de saúde sobre hepatite $B$ em um hospital público do nordeste brasileiro. Rev Bras Saúde Ocup. 2011;36(124):258-64. https://doi.org/10.1590/\$0303-76572011000200009
9. Cortelli AFD. Procedimentos de biossegurança adotados por profissionais prestadores de serviços de manicure, pedicure, tatuagem, piercing e maquiagem definitiva no município de Jacareí, SP [dissertação]. São Paulo: Universidade de São Paulo; 2012[acesso 11 maio 2018]. Disponível em: http://www.teses.usp.br/teses/ disponiveis/6/6135/tde-26102012-114155/pt-br.php

10. Magagnini MAM, Rocha SA, Ayres JA. 0 significado do acidente de trabalho com material biológico para os profissionais de enfermagem. Rev Gaucha Enferm. 2011;32(2):302-8. https://doi.org/10.1590/S1983-14472011000200013

11. Machado MPMS, Duarte LS, Simões LLP, Almeida RPA. Imunidade para hepatite $B$ entre trabalhadores de um hospital de referência em doenças infectocontagiosas. J Health Biol Sci. 2017;5(1):62-70. https://doi. org/10.12662/2317-3076jhbs.v5i1.1018.p62-70.2017

12. Vanzo KLT. Cobertura vacinal e imunidade contra hepatite $B$ em profissionais de saúde da rede pública [dissertação]. Araçatuba: Universidade Estadual Paulista; 2018[acesso 3 dez 2018]. Disponível em: https://repositorio.unesp.br/handle/11449/153285

13. Bittencourt MJS, Miranda MFR, Parijós AM, Mesquita LB, Fonseca DM, Jambo DAA. Dermatofibroma sob pigmento preto de tatuagem: relato de um caso. An Bras Dermatol. 2013;88(4):625-7.

14. Tipple AFV, Mendonça KM, Melo MC, Souza ACS, Pereira MS, Santos SLV. Higienização das mãos: o ensino e a prática entre graduandos na área da saúde. Acta Sci Health Sci. 2007;29(2):107-14. https://doi.org/10.4025/actascihealthsci.1079

15. Anacleto ASCB, Peterline MAS, Pedreira MLG. Higienização das mãos como prática do cuidar: reflexão acerca da responsabilidade profissional. Rev Bras Enferm. 2016;70(2):442-5. https://doi.org/10.1590/0034-7167-2016-0189

16. Rios LRF, Colussi CF. Avaliação normativa dos centros de especialidades odontológicas, Brasil, 2014. Saúde Debate. 2019;43(120):122-36. https://doi.org/10.1590/0103-1104201912009 
17. Vieira-Meyer APGF, Morais APP, Guimarães JMX, Campelo ILB, Vieira NFC, Machado MFAS et al. Infraestrutura e processo de trabalho na atenção primária à saúde: PMAQ no Ceará. Rev Saúde Pública. 2020;54:1-15. https://doi.org/10.11606/s1518-8787.2020054001878

18. Forni IL, Hinny ML, Nardelli A, Consigli C. Alergias a tatuajes y otras reacciones adversas. Alerg Inmunol Clin. 2017;36(1/2):23-9.

19. Miranzi SSC, Gaspar AACS, Iwamoto HH, Miranzi MAS, Dziabas DC. Acidentes de trabalho entre os trabalhadores de uma universidade pública. Rev Bras Saúde Ocup. 2008;33(118):40-7. https://doi.org/10.1590/S0303-76572008000200005

20. Machado MRM, Machado FA. Acidentes com material biológico em trabalhadores de enfermagem do hospital geral de Palmas (TO). Rev Bras Saúde Ocup. 2011;36(124):274-81. https://doi.org/10.1590/S0303-76572011000200011

21. Araújo TME, Silva NC. Acidentes perfurocortantes e medidas preventivas para hepatite $B$ adotadas por profissionais de enfermagem nos serviços de urgência e emergência de Teresina, Piauí. Rev Bras Saúde Ocup. 2014;39(130):175-83. https://doi.org/10.1590/0303-7657000079413

22. Medeiros ED, Gouveia VV, Pimentel CE, Soares AKS, Lima TJS. Escala de atitudes frente a tatuagem: elaboração e evidencias de validade e precisão. Estud Psicol. 2010;27(2):177-86. https://doi.org/10.1590/S0103-166X2010000200005

23. Gouveia VV, Medeiros ED, Mendes LAC, Vione KC, Athayde RAA. Correlatos valorativos de atitudes frente a tatuagem. Psicol Soc. 2010;22(3):476-85. https: / /doi.org/10.1590/S0102-71822010000300008

24. Ministério do Trabalho (BR). Portaria $N^{\circ} 485$, de 11 de novembro de 2005. Aprova a norma regulamentadora $\mathrm{N}^{\circ} 32$ (segurança e saúde no trabalho em estabelecimentos de saúde). Diário Oficial União. 16 nov 2005.

25. Ministério do Trabalho (BR). Norma regulamentadora 6: equipamento de proteção individual EPI. Brasília: Ministério do Trabalho; 1978[acesso 10 set 2020]. Disponível em: https://enit.trabalho.gov.br/portal/images/Arquivos_ SST/SST_NR/NR-06.pdf

26. Angelim RCM, Abrão FMS, Brandão BMGM, Freire DA, Marques SC, Oliveira DC. Práticas de biossegurança de profissionais de saúde nos cuidados ao indivíduo com HIV. Rev Enferm UFSM. 2017;7(3):424-35. https://doi.org/10.5902/2179769225677

27. Vieira M, Padilha MI, Pinheiro RDC. Analysis of accidents with organic material in health workers. Rev Latino-Am Enfermagem. 2011;19(2):1-8. https://doi.org/10.1590/\$0104-11692011000200015

28. Rodrigues RD, Prates TDB, Kramer AM, Rigatto MHP. Manejo da exposição ocupacional a materiais biológicos. Acta Medica. 2016;37(6):1-5.

29. Agência Nacional de Vigilância Sanitária - Anvisa. Resolução RDC № 222, de 28 de março de 2018. Regulamenta as boas práticas de gerenciamento dos resíduos de serviços de saúde, e dá outras providências. Diário Oficial União. 29 mar 2018.

30. Felipe IMA, Silva FMAM, Bassi D, Carvalho AC, Nunes SPH, Dias RS. Biossegurança em centros de embelezamento: estrutura e processamento de materiais. Rev Enferm UFPE. 2019;13:1-10. https://doi.org/10.5205/1981-8963.2019.239171

31. Oliveira D, Novaes RRP, Ferreira WFS, Dutra DA. Gerenciamento de resíduos sólidos em saúde: uma compreensão reflexiva das normativas e aspectos ambientais. Rev Jur Uniandrade. 2020;31(1):1-14.

\section{Agradecimentos}

Os autores agradecem a todos os profissionais da Vigilância Sanitária do município por fornecer as informações do registro dos estabelecimentos, como também a todos os profissionais tatuadores que autorizaram a realização da pesquisa.

\section{Contribuição dos Autores}

Santos BM, Girão MVD - Concepção, planejamento (desenho do estudo), aquisição, análise, interpretação dos dados e redação do trabalho. Arruda FVS - Análise, interpretação dos dados e redação do trabalho. Magalhães MM - Redação do trabalho. Todos os autores aprovaram a versão final do trabalho.

Os autores informam não haver qualquer potencial conflito de interesse com pares e instituições, políticos ou financeiros deste estudo.

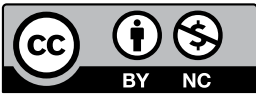

Licença CC BY-NC atribuição não comercial. Com essa licença é permitido acessar, baixar (download), copiar, imprimir, compartilhar, reutilizar e distribuir os artigos, desde que para uso não comercial e com a citação da fonte, conferindo os devidos créditos de autoria e menção à Visa em Debate. Nesses casos, nenhuma permissão é necessária por parte dos autores ou dos editores. 